\title{
TRACE RINGS OF GENERIC MATRICES ARE UNIQUE FACTORIZATION DOMAINS
}

\author{
by LIEVEN LE BRUYN*
}

(Received 3 August, 1984)

Trace rings of generic matrices are U.F.D. A. W. Chatters and D. A. Jordan defined in [0] a unique factorization ring to be a prime ring in which every height one prime ideal is principal. In this note we will prove that the trace ring of $m$ generic $n \times n$-matrices satisfies this condition.

Throughout this note, $k$ will be a field of characteristic zero. Consider the polynomial ring $S=k\left[t_{i j}^{l} ; 1 \leq i, j \leq n, 1 \leq l \leq m\right]$ and the $n \times n$ matrices $X_{l}=\left(t_{i j}^{l}\right)$ in $M_{n}(S)$. The $k$-subalgebra of $M_{n}(S)$ generated by $\left\{X_{l} ; 1 \leq l \leq m\right\}$ is called the ring of $m$ generic $n \times n$ matrices $G_{m, n}$. Adjoining to it the traces of all its elements we obtain the trace ring $\mathbb{T}_{m, n}$ of $m n \times n$ generic matrices, cfr. e.g. [1].

The main aim of this note is to prove:

THEOREM 1. Height one prime ideals of $\mathbb{T}_{m, n}$ are cyclic.

M. Artin and A. Schofield [2] have proved that $\mathbb{T}_{m, n}$ is always a maximal order, cfr. [3]. The following two results liberate their proof from Hilbert-Mumford theory.

LEMMA 2. Let $B$ be a unique factorization domain and $G$ a group of automorphisms of $B$ s.t. $H^{1}\left(G, B^{*}\right)=1$. If $A$ is the fixed ring of $B$ under $G$, then:

(a) $A \subset B$ satisfies no blowing up

(b) $A$ is a unique factorization domain.

Proof. (a) Let $P=B p$ be a height one prime of $B$ s.t. $P \cap A \neq 0$. Then $P$ has a finite orbit under $G$, say $\left\{B p, B p_{1}, \ldots, B p_{k}\right\}$ (take an element $a \in P \cap R$ write $a=p^{k} q_{1}^{l_{1}} \ldots q_{m}^{l_{m}}$, then $\left.\sigma(B p) \in\left\{B p, B q_{i}\right\}\right)$. This shows that for every $\sigma \in G$ there exists a unit $f_{\sigma} \in B^{*}$ s.t. $\sigma\left(p \cdot p_{1} \ldots p_{k}\right)=f_{\sigma} \cdot p p_{1} \ldots p_{k}\left\{f_{\sigma} ; \sigma \in G\right\}$ is clearly a 1 -cocycle, so by assumption there exists a unit $\alpha \in B^{*}$ s.t. $f_{\sigma}=\sigma(\alpha) \alpha^{-1}$ for every $\sigma \in G$. Replace $p$ by $p^{\prime}=\alpha^{-1} \cdot p$, then $p^{\prime} p_{1} \ldots p_{k} \in A$. Therefore, any element $0 \neq \alpha \in P \cap A$ can be written as $a=\left(p^{\prime} p_{1} \ldots p_{k}\right)^{l} q_{l}^{l_{1}} \ldots q_{l}^{l}$, i.e., $a \in\left(p^{\prime} p_{1} \ldots p_{k}\right) A$. So, $P \cap A=\left(p^{\prime} p_{1} \ldots p_{k}\right) A$ and therefore $A \subset B$ satisfies no blowing up (b). By (a) and [4] we know that the natural map $C l(A) \rightarrow C l(B)$ is a homomorphism. Suppose $Q$ is a nonprincipal height one prime of $A$, then $(B Q)^{* *}=B p_{1}^{h_{1}} \ldots p_{m}^{l_{m}}$ for irreducible elements $p_{i} \in B$. Clearly, $Q=B p_{1} \cap A$ which is a principal ideal by the proof of part (a), done.

Let us return to trace rings. Procesi [6] has shown that there exists an action of $P G L_{n}(k)$ on $S$ and $M_{n}(S)$ s.t.

(1) $P G L_{n}(k)$ acts trivially on $k$

(2) The fixed ring of $M_{n}(S)$ under $P G L_{n}(k)$ equals $\mathbb{T}_{m, n}$

(3) The fixed ring of $S$ under $P G L(k)$ equals $R_{m, n}$, the center of $\mathbb{T}_{m, n}$.

* Work supported by an NFWO/FNRS-grant.

Glasgow Math. J. 28 (1986) 11-13. 
COROLlaRY 3. The extension $R_{m, n} \subset S$ satisfies no blowing up and $R_{m, n}$ is a unique factorization domain.

Proof. Because $P G L_{n}(k)$ acts trivially on $k, H^{1}\left(P G L_{n}(k), S^{*}\right)=H^{1}\left(P G L_{n}(k)\right.$, $\left.k^{*}\right)=\operatorname{Hom}\left(P G L_{n}(k), k^{*}\right)=1$ because $P G L_{n}(k)$ is a simple group.

It follows immediately from this result that $\mathbb{J}_{m, n}$ is reflexive as an $R_{m, n}$-module. A reflexive order $\Lambda$ over a normal domain $R$ is said to be a reflexive Azumaya algebra, cfr. e.g. [8], if the natural map:

$$
\phi:\left(\Lambda \otimes_{R} \Lambda^{o p p}\right)^{* *} \rightarrow \operatorname{End}_{R}(\Lambda)
$$

is an isomorphism. It is fairly easy to show that for every divisorial $\Lambda$-ideal $I$ (i.e. a fractional $\Lambda$-ideal which is reflexive as an $R$-module) $I=\Lambda(I \cap R)^{* *}$. We are now in a position to prove Theorem 1 .

Proof of Theorem 1. The proof of the Artin-Schofield theorem shows that the localization of $\mathbb{T}_{m, n}$ at every central height one prime ideal $p$ is an Azumaya algebra (except if $m=n=2$ ). This shows that $\phi_{p}$ is an isomorphism for every $p \in X^{(1)}(R)$. $\mathbb{T}_{m, n}$ being a reflexive $R_{m, n}$-module, this yields that $\mathbb{T}_{m, n}$ is a reflexive Azumaya algebra and the theorem follows from Corollary 3 and the remark above.

If $m=n=2$, then the only height one prime which is not centrally generated is $\mathrm{T}_{2,2}(X Y-Y X)$ which is cyclic, done.

We will give two applications of this result:

THEOREM 4 (Montgomery). Every automorphism of $G_{m, n}$ which leaves the center invariant is the identity.

Proof. By the Skolem-Noether theorem such an automorphism is given by conjugation with a normalizing element of $G_{m, n}, h . G_{m, n} \subset \mathbb{T}_{m, n}$ being a central extension, $h$ is also a normalizing element of $\mathbb{T}_{m, n}$, i.e. $\mathbb{T}_{m, n} . h$ is a divisorial $\mathbb{T}_{m, n}$-ideal.

If $m$ or $n \neq 2$, this entails that $h=\gamma . c$ for some $\gamma \in \mathbb{T}_{m, n}=k$ and $c$ in the field of fractions of $R_{m, n}$, done.

If $m=n=2$, the only noncentral normalizing element of $\mathbb{T}_{m, n}$ is $X Y-Y X$. This element does not normalize $G_{2,2}$, done.

If $\Lambda$ is a maximal order over a normal domain $R$ in some central simple algebra $\Sigma$, we denote by $h(\Lambda)$ the (pointed) set of left $\Lambda$-module isomorphism classes of left fractional $\Lambda$-ideals which are reflexive $R$-modules and with $t_{R}(\Sigma)$ we denote the conjugacy classes of maximal $R$-orders in $\Sigma$.

THEOREM 5. There is a one-to-one correspondence between $h\left(\mathbb{T}_{m, n}\right)$ and $t_{R_{m, n}}\left(Q\left(\mathbb{T}_{m, n}\right)\right)$. ( $m$ and $n$ not both equal to 2 ).

Proof. The correspondence is given by assigning to an (isomorphism class) of a left fractional $\mathbb{T}_{m, n}$-ideal $L$, its right order

$$
O_{r}(L)=\left\{X \in Q\left(\mathbb{T}_{m, n}\right): L x \subset L\right\}
$$


This map is well defined and epimorphic because for any maximal $R_{m, n}$-order in $Q\left(\mathbb{T}_{m, n}\right)$ we can take:

$$
L=\left(\Lambda:, \mathbb{T}_{m, n}\right)=\left\{x \in Q\left(\mathbb{T}_{m, n}\right): x \Lambda \subset \mathbb{T}_{m, n}\right\}
$$

cfr., e.g., [4]. Now suppose $L$ and $L^{\prime}$ are left fractional $\mathbb{T}_{m, n}$-ideals s.t. $O_{r}(L)=$ $\alpha^{-1} . \Theta_{r}\left(L^{\prime}\right) \alpha$, then replacing $L^{\prime}$ by $L^{\prime} \alpha$ we may assume that $\Theta_{r}(L)=\Theta_{r}\left(L^{\prime}\right)=\Lambda$.

Let $M=\left(\left(\Lambda:_{r} \mathbb{T}_{m, n}\right) L\right)^{* *}$ and $M^{\prime}=\left(\left(\Lambda: \mathbb{T}_{m, n}\right) L^{\prime}\right)^{* *}$, then $M$ and $M^{\prime}$ are twosided divisorial $\Lambda$-ideals, i.e., $M=M^{\prime}$. $c$ for some element $c$ in the field of fractions of $R_{m, n}$ (because $\Lambda$ is also a reflexive Azumaya algebra and therefore every twosided divisorial $\Lambda$-ideal is generated by a central element).

Finally

$$
L=\left(\left(\mathbb{T}_{m, n}:{ }_{r} \Lambda\right) M\right)^{* *}=\left(\left(\mathbb{T}_{m, n}:{ }_{r} \Lambda\right) M^{\prime} c\right)^{* *}=L^{\prime} c
$$

finishing the proof.

REMARK. Even if one restricts attention to projective left $\Delta_{m, n}$-ideals, some of them are not free (cfr. [7] in $m=n=2$ case and similarly in $m=3, n=2$ case [5]), so there are maximal orders over $R_{m, n}$ not conjugated to $\mathbb{T}_{m, n}$.

\section{REFERENCES}

0. A. W. Chatters and D. A. Jordan, Non-commutative unique factorization rings, preprint.

1. S. A. Amitsur and Lance W. Small, Prime Ideals in p.i. Rings, J. Algebra 62 (1980), 358-383.

2. Emil Artin, Letter dated December 3rd, 1982.

3. Maurice Auslander and Oscar Goldman, Maximal Orders, Trans. Amer. Math. Soc. 97 (1960), 1-24.

4. Robert M. Fossum, The Divisor Class Group of a Krull Domain, Ergebn. der Math. Wiss. 74 (Springer Verlag, 1973).

5. Lieven Le Bruyn, Homological Properties of Trace Rings of Generic Matrices, Trans. Amer. Math. Soc. (to appear).

6. Claudio Procesi, Invariant Theory of $n \times n$ Matrices, Advances in Math. (1976).

7. Lance W. Small and J. T. Stafford, Holological Properties of Generic Matrices, Israel J. Math. (to appear).

8. Shuen Yuan, Reflexive Modules and Algebra Class Groups over Noetherian Integrally Closed Domains, J. Algebra 32 (1974), 405-417.

Department of Mathematics

UNIVERSITY OF ANTWERP, UIA

UNIVERSITEITSPLEIN 1

2610 WiLRIJK, BELGIUM 